\title{
Hemorragia suprarrenal con hipertensión y excreción aumentada de ácido vanillil mandélico
}

\author{
Drs. M. Eugenia Willshaw Z., * Agostina Olcese D., ** Manuel Neira S., ** Santiago Rubio A. . * Carlos Gutiérrez R. ****
}

\begin{abstract}
We present a rase of unilateral adrenal bemusthage in an infant three onontha old with hypertensiun and inc:reased excrerión of vanillil mandelic acid in twenty four hours urine.

He was operated though radiology was very typical of adrenal hemorrbage in order to exclude a neural creat tumor. We comment this exceptional evilution and the possible explanation.
\end{abstract}

La hemorragia suprarrenal en el periodo de recién nacido puede adoptar fundamentalmente dos formas clínicas. Una con gan compromiso general y signos de shock que tiene pocas expectativas de sobrevida y otra asintomática y que se detecta solamente como hallazgo radiológico posterior. Su frecuencia es baja (en una revisión de 3.637 necropsias se encontraron 43 casos). Presentamos aquí un caso de hemorragia suprartenal unilateral que evolucionó con una masa palpable en el flanco y que por tener cifras tensionales elevadas y un pattern de laboratorio alterado constituyó un problema de diagnóstico diferencial y una conducta exploratoria.

\section{CASO CLINICO}

Lactante de sexo masculino referido a los 3 meses de vida desde Cauquenes por presentar una masa palpable en el flanco izquierdo y que se estimó podia ser un riñón poliquistico. Ingresa en buenas condiciones generales, regular estado nutritivo, signos cutáneos de dermatitis seborreica, supuración del oído derecho.

EJ abdomen se aprecia distendido, higado palpable a $3 \mathrm{~cm}$ del reborde, consistencia normal. En el flanco izquierdo se palpa masa de $5 \times 4 \mathrm{~cm}$, superficie irregular, consistencia aumentada, que no pelotea y que no parece ser sensible.

\footnotetext{
- Servicio Endacrinologia Infantil Hospital Militar

** Servicio de Pediatrí Hospital Militar

*** Servicio de Rayos Hospizal Militar

*t** Servicio de Cimgá Infantil Haspital Militar
}

Entre sus antecedentes perinatales cabe mencionar: $\overline{5}$. " hijo, embarazo de evolución normal, parto espontáneo con período expulsivo rápido, en Hospital de Nueva Imperial. Peso de nacimiento: $4.630 \mathrm{gra}-$ mos y talla de $52 \mathrm{~cm}$. Tuvo ictericia desde el $3 \mathrm{er}$. día de vida, tratada con luminoterapia. Derivado al Hospital Regional de Temuco permanece 15 días y es dado de alta con los diagnósticos de: Recién Nacido a término, grande para edad gestacional, riñón poliquístico, ictericja fisiológica. Se solicita pielografía descendente informada como eliminaciún normal del medio de contraste. Por encima del riñón izquierdo se ve una masa redondeada, calcificada, de límites precisos que parece corresponder a una hemorragia suprarrenal.

En varias oportunidades se constatan cifras tensionales altas que fluctúan entre $120 / 60$ y $160 / 80$. Este hecho sumado al tamaño de la masa palpable ponen en duda el diagnóstico y hacen investigar posibilidad de neuroblastoma.

Se solicitan utros exámenes:

Hemograma: GR: 4.250.000, HTO: $37 \%$, Hb: 12,6 gr\%, G. blancos: 10.900 , caracteres normales. VHS: $11 \mathrm{~mm} / 1$. $^{\text {a hora. }}$

Examen orina: bormal.

Telerradiografía de tôrax: normal.

Acido vanillil-mandélico: $16,38 \mathrm{mg} / \mathrm{gramo}$.

de creatinina: (VN 2,57-6,13)

creatinina: $63,75 \mathrm{mg} / 24 \mathrm{~h}$.

Mielograma: normal

Una 2." determinación de VMA también da valor mayor que el normal ( $11,38 \mathrm{mg} / \mathrm{g}$ de creatinina) para el laboratorio. 
Gráfico 1. Slasu redondeada, calcificada de límites prerisom en regiin suprarrenal izquierda.

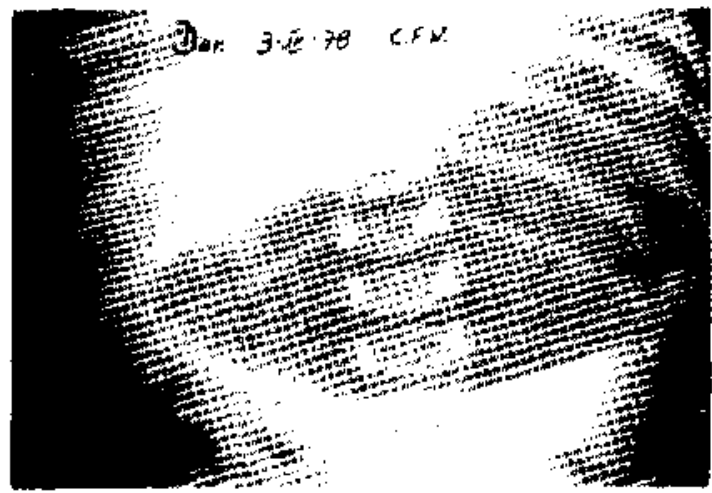

Gráfica 2 y 3. Evolución radiológica de la masa calcificada y que cuincide con lo descrito en une hemorr agja suprarrenal deonatal.
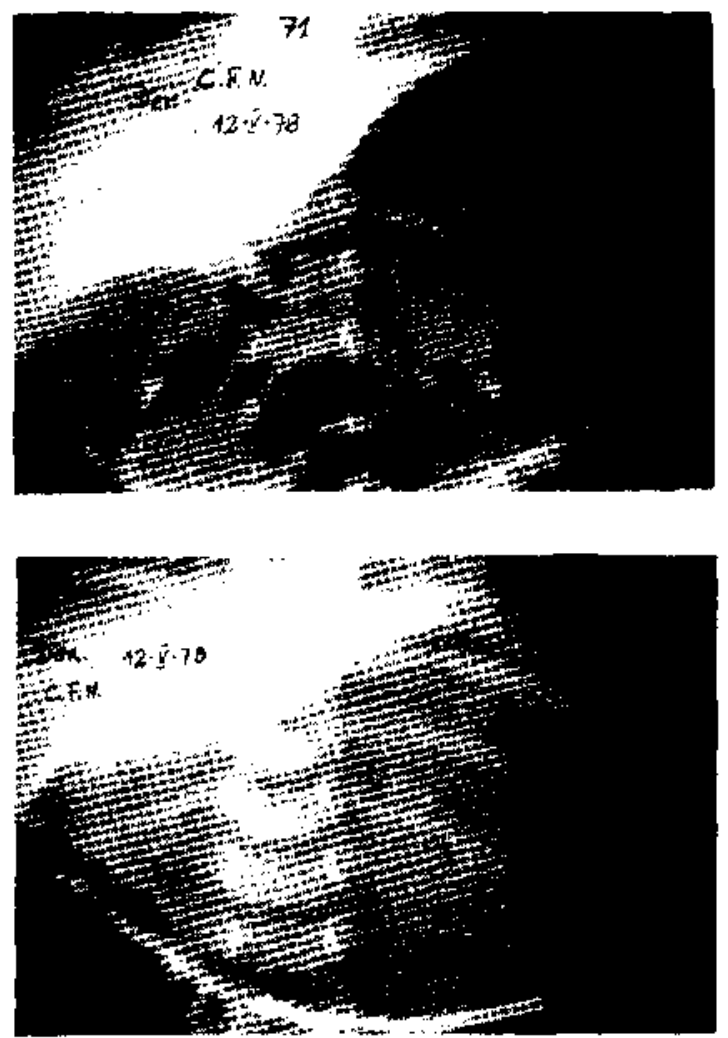

Por la concomitancia de una masa palpable unilateral calcificada de ubicación sobre el riñón, hipertensión y excreción aumentada de ácido vanillil mandélico se decide explorar quirúrgicamente para descartar un tumor de la cresta neural (Neuroblastoma).

En la intervención se encuentra: hígado de aspecto normal, suprarrenal derecha de tamaño y consistencia normal. Suprarrenal izquierda aumentada de tamaño. Adrenalectomía izquierda.
El estudio anatomopatológico revela: trozo ovoideo de $3.5 \mathrm{~cm}$ ell su eje mayor, superficie externa irregular. Se reconoce estructura suprarrenal con un centro de material café rojizo blando, ocupando aparentemente la porción medular de la glándula.

Histológicamente se reconoce glảndula suprarrenal con los 3 componentes. En la zona medular, extenso proceso necrótico hemorrágico en cuya periferia se observan macrófagos cargados de hemosiderina y abundantes depósitos de calcio que sugiere una verdadera barrera; no es posible demostrar existencia tumoral de neuroblastoma.

\section{CONCLUSION}

Hematoma suprarrenal calcificado. Evoluciona bien la presión arterial, se normaliza y es dado de alta a los 6 meses de vida.

\section{DISCUSION}

Este paciente ofrece en su historia clinica hechos frecuentes de observar en una hemorragia suprarrenal y otros excepcionales y que no hemos visto publicados anteriormente en la literatura revisada.

En relación a los hechos habituales podemos diferenciar: los hallazgos clínicos, radiológicos y de laboratorio.

Hechos clínicos habituales observados en este caso: peso alto de nacimiento, parto laboriogo, ictericia de mayor intensidad o mas prolongada, masa palpable en el flanco.

Hallazgos radiológicos típicos: En la 2. semana de vida se presentan al examen radiológico simple, pequeñas calcificaciones agnupadas en forma de mora sobre el polo superior del riñón con un fino halo de intensidad cálcica circunscribiendo el proceso. Controles posteriores revelan regresión parcial de la maqnitud y extensión de las calcificaciones suprarrenales.

Hallazgos de laboratorio: Normalidad de los exámenes de función suprarrenal (salvo compromiso bilateral extenso que lleva a insuficiencia suprarrenal y eliminación de vanillil mandélico normal.

Pese a tener elementos clínicos y radiológicos bastante característicos a favor de hemorragia suprarenal en regresión y con calcificación secundaria, hubo argumentos para plantear otro diagnóstico como el neuroblastoma, fundamentado en la comprobación repetida de presión arterial aumentada y elevación de las cifras de ácido yanillil mandélico por gramo de 
creatinina en 2 oportunidades. Posteriormente al estudio histológico de la masa suprarrenal que permitió descartar neuroblastoma tenemos que tratar de explicar la hipertensión y las alteraciones de laboratono. En cuanto a hipertensión, es posible observarla cuando el tamaño de la masa es de tal magnitud que lleva a una compresión de algún vaso arterial importante o cuando la destrucción de la glándula permite la entrega masiva de esteroides suprarrenales a la circulación, lo que ocurriría en los primeros dias de la hemorragia y no en una etapa tan posterior. Los valores VMA aceptados como normales para lactante son mayores que los consignados en el laboratorio. En una revisión de Gittow y Cols. de 140 niños normales entre un mes y 18 años de edad permitió agruparlos por edad. Estas grandes fluctuaciones er los valores se van minimizando en relación con su avance en edad, logrando igualar prácticamente los valores del adulto alrededor de los 15 años. En el menor de 12 meses las variaciones son extremas siendo su expresión por gramo de creatinina la más adecuada ya que las variaciones diurnas en la excreción de VMA son mínimas. Diapositiva 4.

\section{Tabla 1}

Yalores de excreción de VMA segun edades (de Gitlow y cols.)

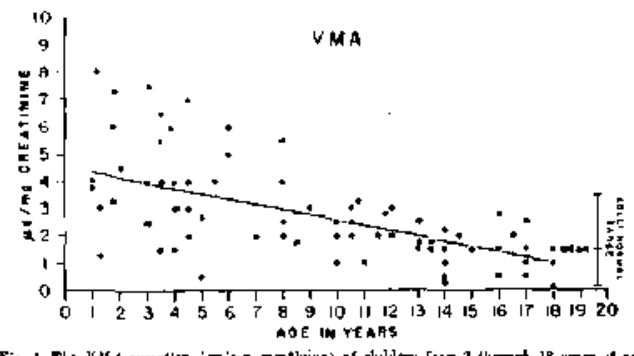

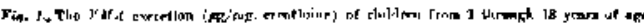

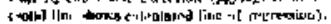

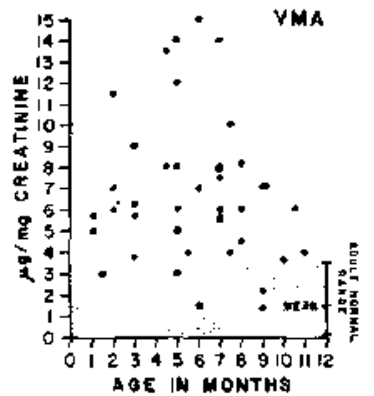

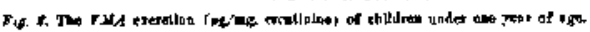

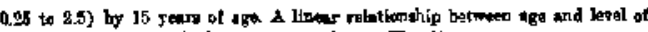

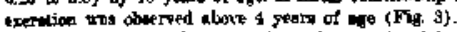

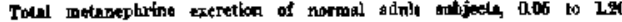

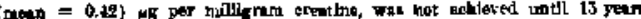

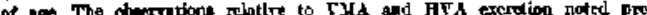

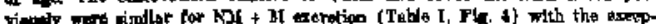

Los valores fluctúan en 15 lactantes menores de 1 año entre 2 y $15 \mathrm{mg} / \mathrm{mg}$ de creatinina. Esta gran variabilidad podría ser la explicación de los valores en nuestros pacientes, especialmente en los primeros meses de la vida, ya que sabemos que la estabilización de los valores y aproximación a las cifras del adulto es un problema de madurez en el tiempo. Está obviada la preparación dietética previa al examen. Existe un caso publicado de coexistencia de neuroblastoma y hemorragia suprarrenal lo que histobógcamente se habría descartado en nuestro paciente.

\section{RESUMEN}

Se presenta un caso de hemorragia suprarrenal unilateral en un lactante de 3 meses que presentó hipertensión y valores mayores de excreción de VMA en orina de $24 \mathrm{~h}$, lo que llevó a la exploración quirúrgica, pese a la radiología altamente sugerente de Hemorragia Suprarrenal para descartar un tumor de la cresta neural. Se descartaron concomitancia de hemorragia suprarrenal y tumor de cresta neural.

\section{AGRADECIMIENTOS}

Al Dr. Hugo Pumarino C. y Laboratorio de Endocrinología del Hospital José Joaquín Aguirre, quien efectuó determinaciones de ácido vanillil mandélico y al Dr. J. J. Latorre (Servicio Anatomía Patológica Hosp. Calvo Mackenna) por el estudio histopatológico correspondiente.

\section{REFERENCIAS}

1 Behrman, R. Neonatel-periuatal medicine. 2." edición. Saint Louig, C. V. Moaby Co., 197 ,

2 Black, J. et al. Natural hislory of adrenal hememorntage in the newborn. Arch. Dis. Child. 48: 183, 1973.

${ }^{3}$ Catel, W. Dingnósjico en pediatria. Salvat Editores, S. A, 1967.

4 Eklof, $O$. Large asymptomatic autrenal haernutomas in the neonate. Acta Radiol. 11: $491,2971$.

${ }^{5}$ Gardiner, $L$ Newer knowledge of adrenocortical disturbances. Ped. Clin. North. Amer. 4: 895, 1957.

${ }^{6}$ Goldzieher, M. et al. The sypdrome hemorthage in the newbors. Vol. 16, N. ${ }^{\circ}$ 2, 1932.

${ }^{7}$ Gross, M. et al. Diegnogis and trealment of neonatal adrenal haemorrhage. J. Pediatr. Sarg- 2: 308, 1967.

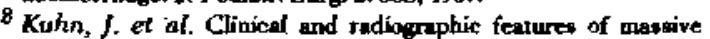
neonatal adrend beeruorrage. Ped. Fadiol. 99: 647, 1971.

9 Pumarino, y atros. Exereción urinaria de VMA en hipertensos esencialea y peuroblentomas. Rev. Méd, Child. 105(8), 1977.

10 Raffensperger. J. et al Abdoninal masses in ehildren under one year of age. Surg. 63: 514, 1968.

11 Rose, J. et al. Massire adrenai heemorrage in newborn. Radiol, 96: 263, 1971 .

12 Steuenson, et al. Adrenal calcification in yoang children. Arch. Dis. Child. 36: 187, 1961.

13 Wagner, A. Bilateral haemorrhagie poeudocyts of the afrenal glands in a newhom. Am. J. Roent. 86: 540, 1961. 